\title{
A Retrospective, Longitudinal, Claims-Based Comparison of Concomitant Diagnoses Between Individuals with and Without Down Syndrome
}

\author{
Amanda M. Kong, MPH; Dana Hurley, PharmD; Kristin A. Evans, PhD; Diana Brixner, PhD; \\ Csilla Csoboth, MD, PhD; and Jeannie Visootsak, MD
}

\begin{abstract}
BACKGROUND: Individuals with Down syndrome (DS) experience various comorbidities in excess of the prevalence seen among the non-DS population. However, the extent of the excess burden of comorbidities specifically within commercially and publicly insured DS populations aged $<21$ years is not currently known.
\end{abstract}

OBJECTIVES: To (a) describe the most common diagnoses among individuals with DS who have either commercial or Medicaid insurance and (b) compare the prevalence of those diagnoses between DS cases and non-DS controls.

METHODS: This was a longitudinal, retrospective study using health care claims of commercially insured and Medicaid-insured individuals in the Truven Health MarketScan Databases from 2008 to 2015. Individuals aged $<2,2-5,6-11$, and 12-20 years with a DS diagnosis (cases; commercial: $n=15,948$; Medicaid: $n=11,958$ ) were matched to individuals without $D S$ (controls; commercial: $n=47,844$; Medicaid: $n=35,874$ ) using a $1: 3$ ratio. The annual number of diagnoses was compared between cases and controls within age groups using t-tests, and the prevalence of the most common diagnoses was compared using chi-square tests.

RESULTS: Cases in all age groups in both databases had more diagnoses annually than controls (mean $=9-17$ per year vs. $4-10$ per year, $P<0.001$ ), and the number of diagnoses decreased with age for cases and controls. Among the most common case diagnoses were upper respiratory infections (28.9\%-59.1\% vs. $19.5 \%-52.9 \%)$; suppurative otitis media (25.1\%-56.8\% vs. $8.7 \%-51.2 \%)$; nutrition/metabolic/developmental symptoms (37.9\%-50.4\% vs. $7.7 \%-10.6 \%)$; delays in development $(22.8 \%-52.8 \%$ vs. $4.1 \%-10.9 \%)$; and general symptoms (35.1\%-47.2\% vs. $22.1 \%-37.2 \%)$, and the prevalence of each was greater among cases versus controls in all age groups in both databases $(P<0.001)$. The most common diagnoses among controls included some of the same as among cases, as well as acute pharyngitis (18.7\%-31.8\% vs. $19.2 \%-30.5 \%)$; allergic rhinitis (19.9\%- $24.3 \%$ vs. $15.3 \%-20.7 \%)$; viral/chlamydial infections (24.2\%-26.6\% vs. $17.7 \%-23.5 \%)$; and joint disorders (11.6\% vs. $16.6 \%)$, and most were significantly more prevalent among cases $(P<0.05)$.

CONCLUSIONS: Commercially insured and Medicaid-insured individuals aged $<21$ years with DS experience a greater number and prevalence of concomitant diagnoses compared with non-DS individuals. Awareness of these common diagnoses could help facilitate the optimal care of these individuals by the pediatric health care community.

J Manag Care Spec Pharm. 2017;23(7):761-70

Copyright $\odot 2017$, Academy of Managed Care Pharmacy. All rights reserved.

\section{What is already known about this subject}

Individuals with Down syndrome (DS) aged $<21$ years are known to experience several comorbid conditions at a greater rate than non-DS individuals, including problems with hearing and vision, heart defects, sleep disturbances, and dementia.

It is recommended that health care providers routinely start early screening for their patients with DS for common comorbidities in order to improve quality of life and life expectancy.

\section{What this study adds}

This study describes the most common diagnoses across the entire health care experience of children and young adults with DS and compares the number and frequency of diagnoses between those with DS and matched controls.

Individuals with DS with commercial and Medicaid insurance are included in the study sample, providing a comparison of the 2 populations that has not been examined to date.

Study results may help payers and case managers proactively manage important concomitant diagnoses common among individuals with DS

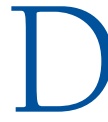
own syndrome (DS) is the most common genetic cause of intellectual disability, accounting for nearly $15 \%$ of cases with a genetic cause. ${ }^{1}$ Individuals with DS experience varying degrees of medical, behavioral, and cognitive symptoms and are at increased risk for Alzheimer's disease and dementia as they age. ${ }^{2-4}$ Although there are currently no approved targeted treatments for DS, early diagnosis and treatment of comorbid medical conditions have contributed to dramatic increases in the life expectancy of individuals with DS. ${ }^{5}$

In 2011, the American Academy of Pediatrics (AAP) released age-specific recommendations for the clinical supervision and assessment of individuals with DS that include screening for specific comorbidities at particular times over the life course. ${ }^{6}$ These guidelines also cite high prevalence of several diagnoses in the DS population, such as hearing and vision problems, sleep disorders, heart defects, and otitis media. In addition, previous studies have identified these and several other conditions as occurring frequently in DS individuals, and some 
studies have also compared the prevalence of certain conditions between DS and non-DS populations. ${ }^{7-10}$ However, no study to date has taken a comprehensive look at all of the diagnoses received by individuals with DS in a health care setting and compared the frequency of those diagnoses with individuals who do not have DS.

The purpose of this study was to describe the number and type of concomitant diagnoses observed in 2 large health care claims databases of commercially insured and Medicaidinsured children, adolescents, and young adults (aged $<21$ years) with DS. This study also aimed to compare the prevalence of common diagnoses in this population between individuals with DS and individuals who do not have DS.

\section{Methods}

\section{Data Sources}

This was a longitudinal, retrospective cohort study using the health care claims of individuals with DS (cases) and without DS (controls) contained in the Truven Health MarketScan Commercial Claims and Encounters (commercial) and Truven Health Medicaid Multi-State (Medicaid) Databases. The commercial database is a large, primarily employer-based health care claims database that includes inpatient admissions, outpatient visits, and outpatient prescription drug claims of employees and their dependents covered under various types of commercial health care plans. The Medicaid database contains the inpatient admissions, outpatient visits, and outpatient prescription drug claims of Medicaid enrollees from approximately 10 geographically dispersed states across the United States. Because of data agreements, the specific states represented in the Medicaid database cannot be reported. Managed and fee-for-service plans are included.

Both databases satisfy the conditions set forth in Sections 164.514 (a)-(b)lii of the Health Insurance Portability and Accountability Act of 1996 privacy rule regarding the determination and documentation of statistically de-identified data. Because this study used only de-identified patient records and did not involve the collection, use, or transmittal of individually identifiable data, institutional review board approval to conduct this study was not required.

\section{Patient Selection}

The sample of DS cases for this study was drawn from all individuals in the commercial and Medicaid databases who had at least 1 nondiagnostic medical claim (i.e., not a laboratory or radiology claim) with an International Classification of Diseases, Ninth Revision, Clinical Modification (ICD-9-CM) diagnosis code for DS (758.0x) between January 1, 2008, and December 31,2014 . Individuals with DS who were aged $<21$ years on January 1 of the year of their first qualifying DS diagnosis (index year) and who were continuously enrolled in their insurance plans from January 1 through December 31 of that year were included in the analysis. We focused on this population to highlight common non-DS conditions among the pediatric population aged $<21$ years who are likely to experience different conditions than older DS populations. The control sample without DS was drawn from all individuals in each respective database who had no claims containing a diagnosis of DS or any other chromosomal anomaly (ICD-9-CM 758.1x-758.9x) between January 1, 2008, and December 31, 2014. All cases and controls were followed for a minimum of 12 months. The evaluation period (follow-up time) for each case began with January 1 of their index year and ended with December 31 of the last consecutive full calendar year during which they were continuously enrolled and aged $<21$ years on January 1 of that year.

Using an exact match, each DS case was then matched to 3 controls of the same age (on January 1 of the case's index year), sex, type of insurance plan, and number of consecutive full calendar years in the evaluation period. Also, cases and controls in the commercial sample were matched on geographic region (only available in the commercial data), and Medicaid cases and controls were matched on state of residence and race (only available in the Medicaid data). The 3 controls included in the analyses for a given case were randomly selected from all controls within the respective database that met all matching criteria for that case.

\section{Variables}

The main independent variable examined in this analysis was the presence of a DS diagnosis (i.e., cases vs. controls). Comparisons were also made between clinically relevant age groups $(<2,2-5,6-11$, and 12-20 years) and between commercial and Medicaid cases. These age groups were chosen based on key periods for growth and human development: infancy ( $<2$ years), early childhood ( $2-5$ years), middle childhood (6-11 years), and adolescence/young adulthood (12-20 years).

The following 3 outcome variables were measured: (1) annual number of diagnoses per patient; (2) most common diagnoses among the combined sample (commercial and Medicaid) of cases and separately for cases in each database; and (3) most common diagnoses in the combined sample (commercial and Medicaid) of controls. All diagnoses were captured at the 3-digit level of the ICD-9-CM classification system. Primary and secondary diagnoses on inpatient and outpatient claims were evaluated.

Each outcome variable was assessed for each calendar year of an individual's evaluation period, and individuals could contribute data to multiple age groups if they crossed into subsequent age groups during their evaluation periods. The number of diagnoses was annualized for each individual within the age groups included in their evaluation period, and the presence of each diagnosis of interest was assessed across all calendar years in a given age group for each individual. For example, 
TABLE 1 Demographic Characteristics at Index of Commercial and Medicaid Individuals with Down Syndrome (Cases) and Without Down Syndrome (Controls)

\begin{tabular}{|c|c|c|c|c|c|c|c|c|}
\hline \multirow[b]{4}{*}{ Age at index, mean (SD) } & \multicolumn{4}{|c|}{ Commercial } & \multicolumn{4}{|c|}{ Medicaid } \\
\hline & \multicolumn{2}{|c|}{ Cases } & \multicolumn{2}{|c|}{ Controls } & \multicolumn{2}{|c|}{ Cases } & \multicolumn{2}{|c|}{ Controls } \\
\hline & \multicolumn{2}{|c|}{$\mathrm{n}=15,948$} & \multicolumn{2}{|c|}{$\mathrm{n}=47,844$} & \multicolumn{2}{|c|}{$\mathrm{n}=11,958$} & \multicolumn{2}{|c|}{$\mathrm{n}=35,874$} \\
\hline & 8.7 & $(6.0)$ & 8.7 & $(6.0)$ & 8.1 & $(6.1)$ & 8.1 & $(6.1)$ \\
\hline \multicolumn{9}{|l|}{ Age group at index, years, \% (n) } \\
\hline$<2$ & 13.7 & $(2,180)$ & 13.7 & $(6,540)$ & 17.1 & $(2,047)$ & 17.1 & $(6,141)$ \\
\hline $2-5$ & 23.0 & $(3,661)$ & 23.0 & 10,983 & 23.9 & $(2,854)$ & 23.9 & $(8,562)$ \\
\hline $6-11$ & 29.3 & $(4,671)$ & 29.3 & $(14,013)$ & 28.5 & $(3,412)$ & 28.5 & $(10,236)$ \\
\hline $12-20$ & 34.1 & $(5,436)$ & 34.1 & $(16,308)$ & 30.5 & $(3,645)$ & 30.5 & $(10,935)$ \\
\hline Male, \% (n) & 53.6 & $(8,556)$ & 53.6 & $(25,668)$ & 54.4 & $(6,505)$ & 54.4 & $(19,515)$ \\
\hline \multicolumn{9}{|l|}{ Race/ethnicity, \% (n)a } \\
\hline White & & - & & - & 49.2 & $(5,879)$ & 49.2 & $(17,637)$ \\
\hline Black & & - & & - & 15.5 & $(1,852)$ & 15.5 & $(5,556)$ \\
\hline Hispanic & & - & & - & 7.5 & $(900)$ & 7.5 & $(2,700)$ \\
\hline Other & & - & & - & 27.8 & $(3,327)$ & 27.8 & $(9,981)$ \\
\hline \multicolumn{9}{|l|}{ Geographic region, \% (n)a } \\
\hline Northeast & 17.0 & $(2,709)$ & 17.0 & $(8,127)$ & & - & & - \\
\hline North Central & 28.5 & $(4,540)$ & 28.5 & $(13,620)$ & & - & & - \\
\hline South & 35.6 & $(5,677)$ & 35.6 & $(17,031)$ & & - & & - \\
\hline West & 16.9 & $(2,689)$ & 16.9 & $(8,067)$ & & - & & - \\
\hline Unknown & 2.1 & (333) & 2.1 & (999) & & - & & - \\
\hline \multicolumn{9}{|l|}{ Insurance plan type, \% (n) } \\
\hline Comprehensive/indemnity & 1.1 & (176) & 1.1 & $(528)$ & 53.5 & $(6,397)$ & 53.5 & $(19,191)$ \\
\hline EPO & 1.1 & (183) & 1.1 & $(549)$ & 0.0 & (0) & 0.0 & $(0)$ \\
\hline POS with or without capitation & 7.1 & $(1,122)$ & 7.1 & $(3,366)$ & 13.4 & $(1,598)$ & 13.4 & $(4,794)$ \\
\hline $\mathrm{HMO}$ & 14.0 & $(2,235)$ & 14.0 & $(6,705)$ & 27.8 & $(3,320)$ & 27.8 & $(9,960)$ \\
\hline $\mathrm{PPO}$ & 62.2 & $(9,923)$ & 62.2 & $(29,769)$ & 0.0 & $(0)$ & 0.0 & $(0)$ \\
\hline CDHP/HDHP & 7.7 & $(1,225)$ & 7.7 & $(3,675)$ & 0.0 & $(0)$ & 0.0 & $(0)$ \\
\hline Unknown & 6.8 & $(1,084)$ & 6.8 & $(3,252)$ & 5.4 & $(643)$ & 5.4 & $(1,929)$ \\
\hline \multicolumn{9}{|l|}{ Years of evaluation period } \\
\hline Minimum & & 1 & & 1 & & 1 & & 1 \\
\hline Median & & 2 & & 2 & & 3 & & 3 \\
\hline Maximum & & 7 & & 7 & & 7 & & 7 \\
\hline
\end{tabular}

if an individual was age 3 years at index and the evaluation period was 5 years long, he or she contributed 3 years of data (ages 3,4 , and 5 years) to the 2-5 age group and 2 years of data (ages 6 and 7 years) to the 6-11 age group. The total number of diagnoses was then quantified within each of those calendar years separately, and an age group average was calculated for that individual for each of the 2 age groups. The individual would also be assessed for the presence of the most common diagnoses among those aged 2-5 years any time while in that age group and for the most common diagnoses among those aged 6-11 years while in that age group. Statistical comparisons were made with t-tests and chi-square tests for continuous and categorical variables, respectively. $P$ values $<0.05$ were considered statistically significant. Analyses were conducted with SAS version 9.3 (SAS Institute, Cary, NC).

\section{Results}

A total of 15,948 qualifying individuals with DS were matched to 47,844 controls in the commercial database, and 11,958 qualifying DS cases were matched to 35,874 controls in the Medicaid database (Appendix A, available in online article). Commercial cases and controls were slightly older than Medicaid cases and controls (mean [standard deviation] age $=8.7[6.0]$ vs. $8.1[6.1]$ ), and more than half of all cases and controls in each cohort were male. Evaluation periods in the commercial and Medicaid cohorts ranged from 1 to 7 years long, with median follow-up times of 2 and 3 years in the commercial and Medicaid cohorts, respectively (Table 1).

Across all age groups in both databases, cases had significantly more diagnoses than controls, with the annual average 


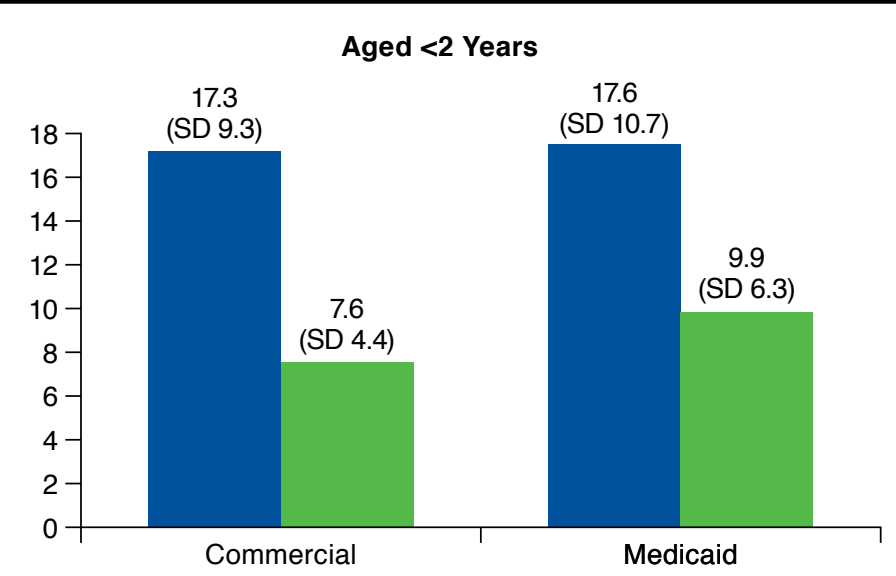

Aged 2-5 Years
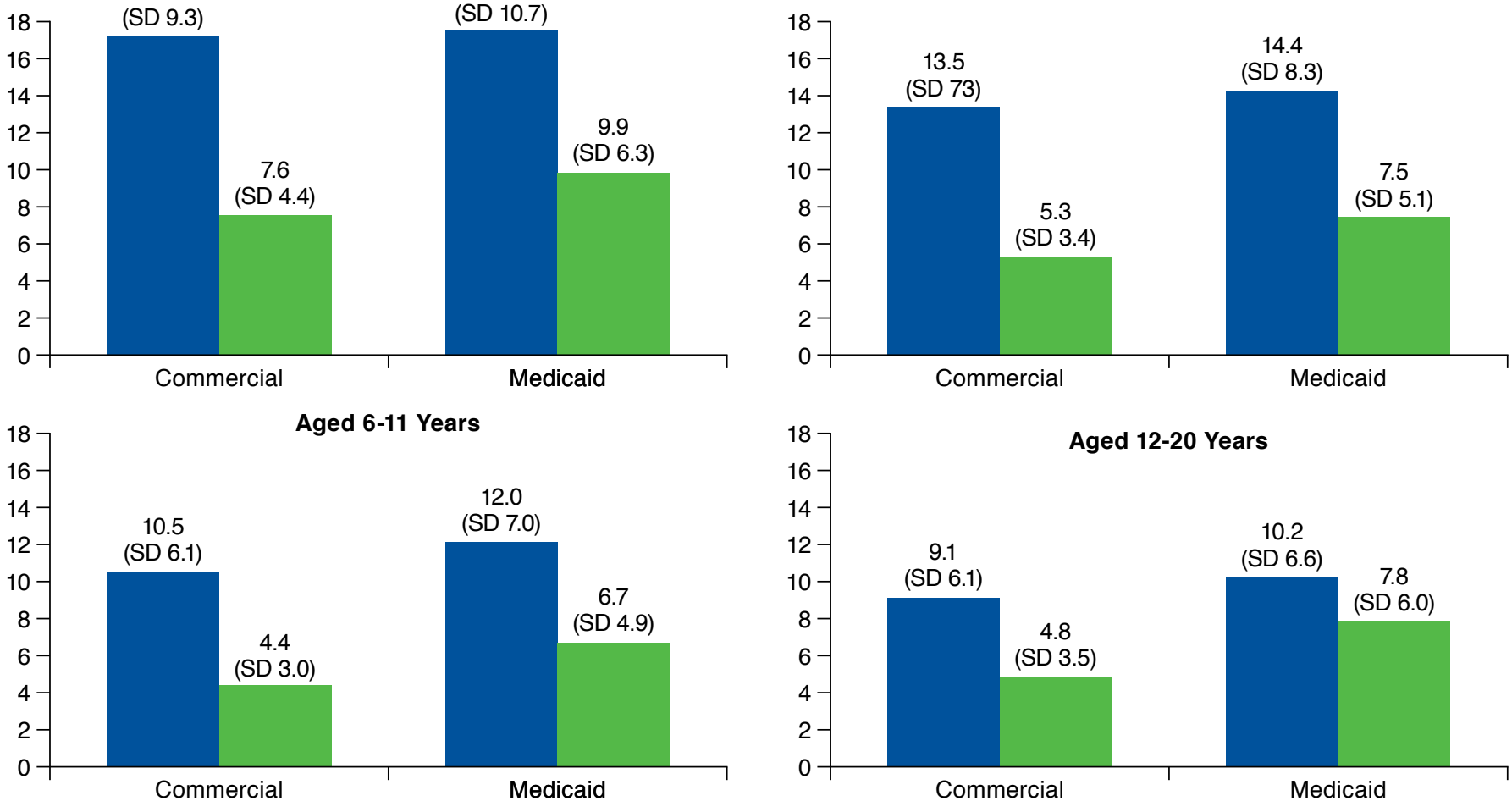

Individuals with DS Individuals without DS

Note: $P<0.001$ for $t$-tests for comparisons between individuals with DS and individuals without DS in the 2 payer groups and for comparisons between individuals with DS and without DS with commercial insurance and individuals with DS and without DS with Medicare insurance.

DS = Down syndrome; $S D=$ standard deviation

number of diagnoses decreasing with age (Figure 1). The youngest DS cases in the commercial and Medicaid cohorts had a similar number of diagnoses, but older Medicaid cases had significantly more diagnoses annually than older commercial cases $(P<0.001)$. Also, Medicaid controls had 30\%-60\% more diagnoses annually, compared with commercial controls $(P<0.001)$.

Figure 2 presents the most common case diagnoses by age in the combined commercial and Medicaid sample. The top 5 diagnoses in each age group were identified, but 8 diagnoses are represented in Figure 2 because of differences in the top diagnoses between age groups. Acute upper respiratory infections and suppurative otitis media were among the 5 most common diagnoses in all age groups for cases and controls, with a small but significant difference between those cases and controls aged $<2$ years and greater differences in the older age groups $(P<0.001)$.
The largest differences in prevalence were observed for symptoms concerning nutrition, metabolism, and development (e.g., abnormal weight gain/loss, feeding difficulties, anorexia, and polydipsia) and specific delays in development.

Table 2 compares the combined sample of cases and controls for the frequency of the 10 most common diagnoses among cases in each age group. In addition to the diagnoses shown in Figure 2, certain congenital heart anomalies, hearing loss, and digestive symptoms were commonly diagnosed among DS cases for most age groups. The prevalence of common case diagnoses was generally higher for Medicaid cases of all ages compared with commercial cases, including symptoms concerning nutrition, metabolism, and development (Medicaid: 21.7\%-54.0\% vs. commercial: $12.8 \%-46.9 \% ; P<0.001$ ); general symptoms (32.0\%-51.7\% vs. 39.2\%-43.5\%; $P<0.001)$; respiratory/chest symptoms (29.3\%-46.7\% vs. $25.1 \%-33.3 \% ; P=0.024$ 


\section{FIGURE 2 Proportion of All Individuals with and Without Down Syndrome (Commercial and Medicaid} Combined) in Each Age Group with the Most Common 3-Digit ICD-9-CM Diagnoses Among Individuals with Down Syndrome
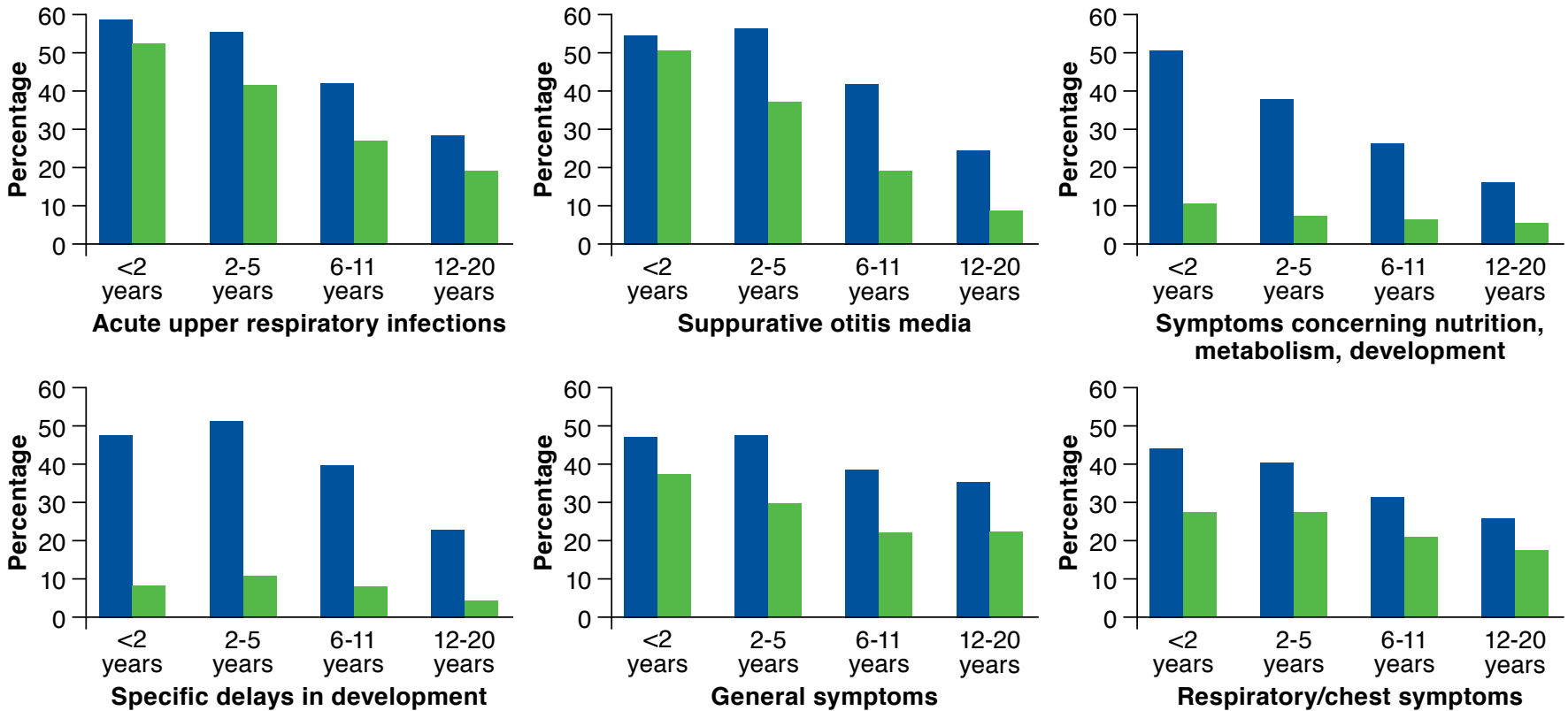

Specific delays in development
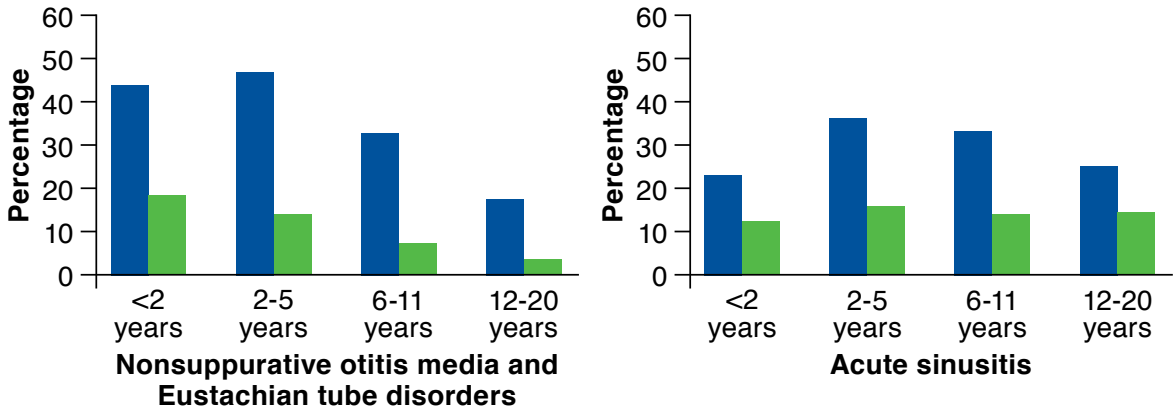

Individuals with DS

Indvidiuals without DS

Note: $P<0.001$ for chi-squre tests for comparisons between individuals with and without DS in each age group for each condition except the comparison of respiratory/chest symptoms in the age group 6-11 years, where $P=0.008$.

DS = Down syndrome.

for the age group $<2$ years and $P<0.001$ for age groups $2-5$, 6-11, and 12-20); and specific delays in development (36.8\%$68.0 \%$ vs. $12.2 \%-39.9 \% ; P<0.001$; data not shown). Acute sinusitis was diagnosed more frequently among commercial cases (Medicaid: 20.5\%-34.3\% vs. commercial: 26.8\%-40.7\%; $P<0.001$ for age groups $<2,2-5$, and $6-11$, and $P=0.036$ for the 12-20 age group; data not shown).

Figure 3 shows the differences between the combined sample of commercial and Medicaid cases and the combined sample of controls for the frequency of the 5 most common diagnoses among controls in each age group. The most common control diagnoses were similar to those most commonly observed among cases. Despite these being the most commonly observed diagnoses in controls, in general, they were significantly more frequent among cases for all age groups (upper respiratory infections, allergic rhinitis, general symptoms, suppurative otitis media, and respiratory/chest symptoms; $P<0.001$ ). Appendix B (available in online article) includes the 10 most common diagnoses in each age group in the combined sample of commercial and Medicaid controls. In addition to the diagnoses shown in Figure 3, digestive symptoms were commonly diagnosed among most age groups; disorders of the conjunctiva were among the most common diagnoses for controls in the 2 younger age groups; and asthma was common 


\section{A Retrospective, Longitudinal, Claims-Based Comparison of Concomitant Diagnoses Between Individuals with and Without Down Syndrome}

\section{TABLE 2 Number and Proportion of All Individuals with and Without Down Syndrome /Commercial and Medicaid Combined) with the 10 Most Common 3-Digit ICD-9-CM Diagnoses Among Individuals with Down Syndrome in Each Age Group}

\begin{tabular}{|c|c|c|c|c|c|}
\hline Most Common 3-Digit ICD-9-CM Diagnoses Among Cases During Follow-up ${ }^{\mathrm{a}}$ & \multicolumn{2}{|c|}{ Cases $^{\mathrm{b}}$} & \multicolumn{2}{|c|}{ Controls ${ }^{c}$} & $\begin{array}{l}P \text { Values from } \\
\text { Chi-Square Test }\end{array}$ \\
\hline \multirow{2}{*}{ Aged $<2$ Years } & \multicolumn{2}{|c|}{$\mathrm{n}=4,227$} & \multicolumn{2}{|c|}{$\mathrm{n}=12,661$} & \\
\hline & $\%$ & $\mathrm{n}$ & $\%$ & $\mathbf{n}$ & \\
\hline Acute upper respiratory infections of multiple or unspecified sites (465.xx) & 59.1 & $(2,499)$ & 52.9 & $(6,705)$ & $<0.001$ \\
\hline Suppurative and unspecified otitis media (382.xx) & 55.1 & $(2,327)$ & 51.2 & $(6,485)$ & $<0.001$ \\
\hline Symptoms concerning nutrition, metabolism, and development (783.xx) & 50.4 & $(2,129)$ & 10.6 & $(1,338)$ & $<0.001$ \\
\hline Specific delays in development (315.xx) & 48.9 & $(2,066)$ & 8.4 & $(1,061)$ & $<0.001$ \\
\hline General symptoms (e.g., fatigue, fever, insomnia, syncope; 780.xx) & 46.8 & $(1,980)$ & 37.2 & $(4,709)$ & $<0.001$ \\
\hline Symptoms involving respiratory system and other chest symptoms (786.xx) & 44.9 & $(1,899)$ & 28.6 & $(3,625)$ & $<0.001$ \\
\hline Nonsuppurative otitis media and Eustachian tube disorders (381.xx) & 44.7 & $(1,889)$ & 19.2 & $(2,434)$ & $<0.001$ \\
\hline Bulbus cordis anomalies and anomalies of cardiac septal closure (745.xx) & 42.9 & $(1,815)$ & 1.6 & $(204)$ & $<0.001$ \\
\hline Symptoms involving digestive system (787.xx) & 37.1 & $(1,567)$ & 20.8 & $(2,636)$ & $<0.001$ \\
\hline Hearing loss (389.xx) & 34.3 & $(1,451)$ & 4.2 & $(538)$ & $<0.001$ \\
\hline Acute bronchitis and bronchiolitis (466.xx) & 32.0 & $(1,352)$ & 19.6 & $(2,487)$ & $<0.001$ \\
\hline Acute sinusitis (461.xx) & 24.5 & $(1,035)$ & 12.9 & $(1,639)$ & $<0.001$ \\
\hline \multirow{2}{*}{ Aged 2-5 Years } & \multicolumn{2}{|c|}{$\mathbf{n}=8,955$} & \multicolumn{2}{|c|}{$\mathrm{n}=26,865$} & \\
\hline & $\%$ & $\mathbf{n}$ & $\%$ & $\mathrm{n}$ & \\
\hline Suppurative and unspecified otitis media (382.xx) & 56.8 & $(5,089)$ & 37.5 & $(10,081)$ & $<0.001$ \\
\hline Acute upper respiratory infections of multiple or unspecified sites (465.xx) & 56.0 & $(5,012)$ & 42.0 & $(11,272)$ & $<0.001$ \\
\hline Specific delays in development (315.xx) & 52.8 & $(4,728)$ & 10.9 & $(2,929)$ & $<0.001$ \\
\hline Nonsuppurative otitis media and Eustachian tube disorders (381.xx) & 48.4 & $(4,336)$ & 14.5 & $(3,900)$ & $<0.001$ \\
\hline General symptoms (e.g., fatigue, fever, insomnia, syncope; 780.xx) & 47.2 & $(4,230)$ & 29.9 & $(8,029)$ & $<0.001$ \\
\hline Symptoms involving respiratory system and other chest symptoms (786.xx) & 41.9 & $(3,755)$ & 28.1 & $(7,539)$ & $<0.001$ \\
\hline Symptoms concerning nutrition, metabolism, and development (783.xx) & 37.9 & $(3,393)$ & 7.7 & $(2,076)$ & $<0.001$ \\
\hline Acute sinusitis (461.xx) & 37.8 & $(3,385)$ & 16.0 & $(4,292)$ & $<0.001$ \\
\hline Symptoms involving digestive system (787.xx) & 36.4 & $(3,257)$ & 16.2 & $(4,354)$ & $<0.001$ \\
\hline Hearing loss (389.xx) & 32.6 & $(2,923)$ & 5.0 & $(1,346)$ & $<0.001$ \\
\hline Bulbus cordis anomalies and anomalies of cardiac septal closure (745.xx) & 29.4 & $(2,635)$ & 0.9 & $(251)$ & $<0.001$ \\
\hline Disorders of refraction and accommodation (367.xx) & 24.0 & $(2,146)$ & 8.2 & $(2,206)$ & $<0.001$ \\
\hline \multirow{2}{*}{ Aged 6-11 Years } & \multicolumn{2}{|c|}{$n=11,810$} & \multicolumn{2}{|c|}{$\mathbf{n}=35,430$} & \\
\hline & $\%$ & $\mathrm{n}$ & $\%$ & $\mathrm{n}$ & \\
\hline Suppurative and unspecified otitis media (382.xx) & 42.4 & $(5,012)$ & 19.6 & $(6,939)$ & $<0.001$ \\
\hline Acute upper respiratory infections of multiple or unspecified sites (465.xx) & 42.4 & $(5,003)$ & 27.4 & $(9,719)$ & $<0.001$ \\
\hline General symptoms (e.g., fatigue fever, insomnia, syncope; 780.xx) & 38.3 & $(4,525)$ & 22.1 & $(7,829)$ & $<0.001$ \\
\hline Acute sinusitis (461.xx) & 34.9 & $(4,116)$ & 14.4 & $(5,090)$ & $<0.001$ \\
\hline Nonsuppurative otitis media and Eustachian tube disorders (381.xx) & 33.7 & $(3,977)$ & 7.5 & $(2,644)$ & $<0.001$ \\
\hline Symptoms involving respiratory system and other chest symptoms (786.xx) & 32.7 & $(3,860)$ & 21.6 & $(7,646)$ & 0.008 \\
\hline Acute pharyngitis (462.xx) & 31.8 & $(3,757)$ & 30.5 & $(10,808)$ & $<0.001$ \\
\hline Symptoms involving digestive system (787.xx) & 27.3 & $(3,226)$ & 12.4 & $(4,401)$ & $<0.001$ \\
\hline Disorders of external ear (380.xx) & 26.2 & $(3,099)$ & 9.5 & $(3,360)$ & $<0.001$ \\
\hline Hearing loss (389.xx) & 26.2 & $(3,093)$ & 3.2 & $(1,148)$ & $<0.001$ \\
\hline Strabismus and other disorders of binocular eye movements (378.xx) & 24.8 & $(2,933)$ & 3.3 & $(1,180)$ & $<0.001$ \\
\hline \multirow{2}{*}{ Aged 12-20 Years } & \multicolumn{2}{|c|}{$\mathrm{n}=11,984$} & \multicolumn{2}{|c|}{$\mathrm{n}=35,952$} & \\
\hline & $\%$ & $\mathrm{n}$ & $\%$ & $\mathbf{n}$ & \\
\hline General symptoms (e.g., fatigue, fever, insomnia, syncope; 780.xx) & 35.1 & $(4,209)$ & 22.1 & $(7,932)$ & $<0.001$ \\
\hline Acute upper respiratory infections of multiple or unspecified sites (465.xx) & 28.9 & $(3,462)$ & 19.5 & $(7,018)$ & $<0.001$ \\
\hline Symptoms involving respiratory system and other chest symptoms (786.xx) & 26.9 & $(3,219)$ & 18.4 & $(6,613)$ & $<0.001$ \\
\hline Acute sinusitis (461.xx) & 26.1 & $(3,124)$ & 15.1 & $(5,418)$ & $<0.001$ \\
\hline Suppurative and unspecified otitis media (382.xx) & 25.0 & $(2,992)$ & 8.7 & $(3,139)$ & $<0.001$ \\
\hline Disorders of external ear (380.xx) & 24.6 & $(2,945)$ & 6.3 & $(2,274)$ & $<0.001$ \\
\hline Acquired hypothyroidism (244.xx) & 23.9 & $(2,859)$ & 1.3 & $(463)$ & $<0.001$ \\
\hline
\end{tabular}




\section{TABLE 2 Number and Proportion of All Individuals with and Without Down Syndrome /Commercial and Medicaid Combined) with the 10 Most Common 3-Digit ICD-9-CM Diagnoses Among Individuals with Down Syndrome in Each Age Group (continued)}

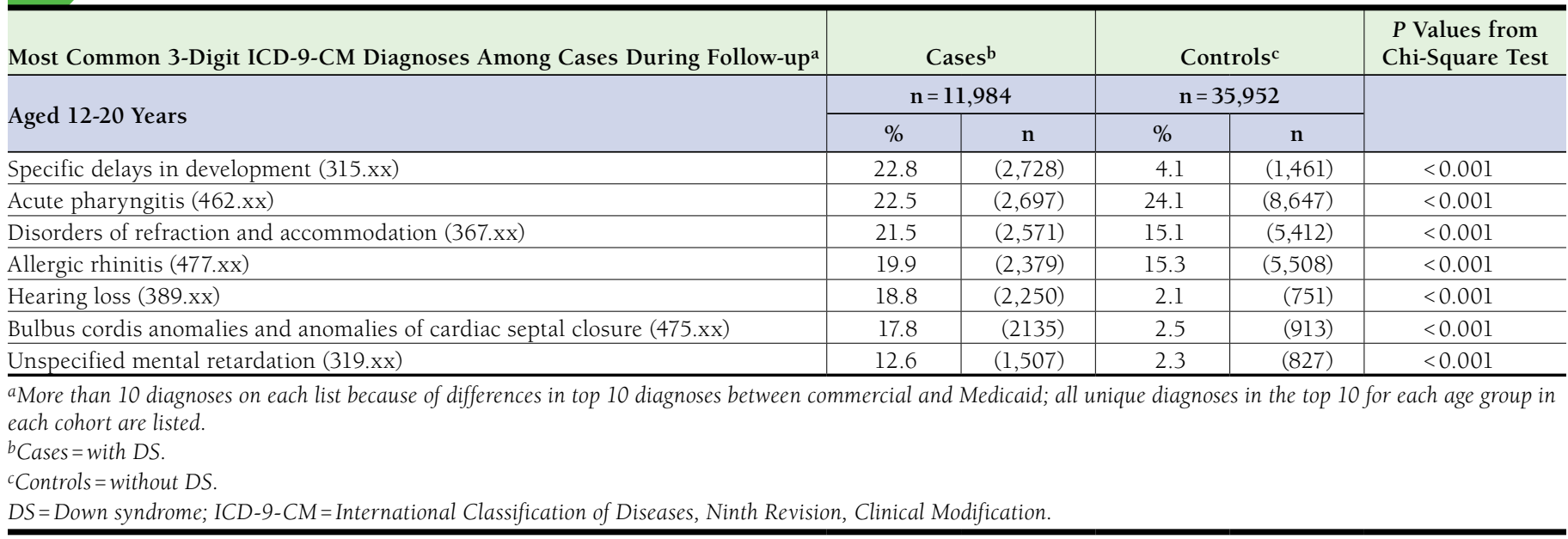

among controls aged 2-11 years, although the prevalence for these conditions was higher for the cases cohort.

\section{Discussion}

In this retrospective claims-based study, individuals with DS aged $<21$ years experienced substantially greater prevalence of specific common concomitant diagnoses, compared with similar individuals without DS. Certain respiratory ailments, ear infections, and developmental conditions were among the most frequent diagnoses in DS cases, and even the most common conditions among controls were often more prevalent among cases (e.g., upper respiratory infections and suppurative otitis media). In addition, DS cases received from $30 \%$ to $>100 \%$ as many annual diagnoses during follow-up, compared with nonDS controls. We also observed lower rates of hearing loss in the DS population than expected.

Several of the diagnoses observed among DS individuals in this study are among those mentioned in the AAP guidelines for health care supervision of children with DS, such as respiratory conditions and congenital heart disease. ${ }^{6}$ In this study, acute upper respiratory infections, bronchitis/bronchiolitis, and other respiratory and chest symptoms were diagnosed significantly more frequently in DS cases than controls, which is consistent with other reports of higher incidence of various respiratory conditions among individuals with DS. ${ }^{7,11}$ For example, Schieve et al. (2009) observed $>100 \%$ increased odds of respiratory allergies among DS children aged 3-10 years $(P<0.05),{ }^{7}$ compared with those without DS. Stagliano et al. (2015) reported a $>200 \%$ increased risk of hospitalization for respiratory syncytial virus associated with a DS diagnosis among children aged 0-3 years $(P<0.05) \cdot{ }^{11}$ In addition, several studies have reported respiratory diagnoses to be among the most common infections and causes of hospitalization among individuals with DS. ${ }^{12}$

The AAP guidelines also recommend that infants with DS between birth and 1 month of age be screened for congenital heart defects, given prevalence estimates of approximately $40 \%-55 \%$ in this population. ${ }^{6,13-16}$ In line with these estimates and other previous work, ${ }^{9,17}$ up to $47 \%$ of DS cases in the current study received a diagnosis of "bulbus cordis anomalies and anomalies of cardiac septal closure," with the highest frequency among DS cases aged $<2$ years with commercial insurance. The decreasing frequency of heart anomaly diagnoses across increasing age groups may be a result of the practice of performing echocardiogram testing for all newborns with DS, which generally leads to the diagnosis of congenital heart defects early in life. Fewer heart defect diagnoses in the older age groups may be a result of the conditions becoming stable with age and subsequent undercoding of them by physicians or, as suggested by Boulet et al. (2008), ${ }^{17}$ early deaths from heart defects and/or surgical corrections of the conditions early in life.

The current results are also consistent with the findings of Zhu et al. (2013) regarding hospitalizations for various causes among individuals with DS. ${ }^{18}$ In that study, DS cases were more than twice as likely as non-DS controls to be hospitalized, with the greatest admission rate ratios observed for congenital heart defects and other malformations, gastrointestinal defects, nervous system and sensory disorders, and respiratory conditions. Although inpatient admissions were not specifically examined in the current study, congenital heart defects were diagnosed up to 33 times as often; eye and ear disorders (e.g., hearing loss and refraction/accommodation disorders) up to 9 times as often; respiratory ailments (e.g., acute upper respiratory infections and acute sinusitis) up to 2.4 times as often; and 


\section{FIGURE 3 Proportion of All Individuals with and Without Down Syndrome (Commercial and Medicaid} Combined) in Each Age Group with the Most Common 3-Digit ICD-9-CM Diagnoses Among Individuals Without Down Syndrome
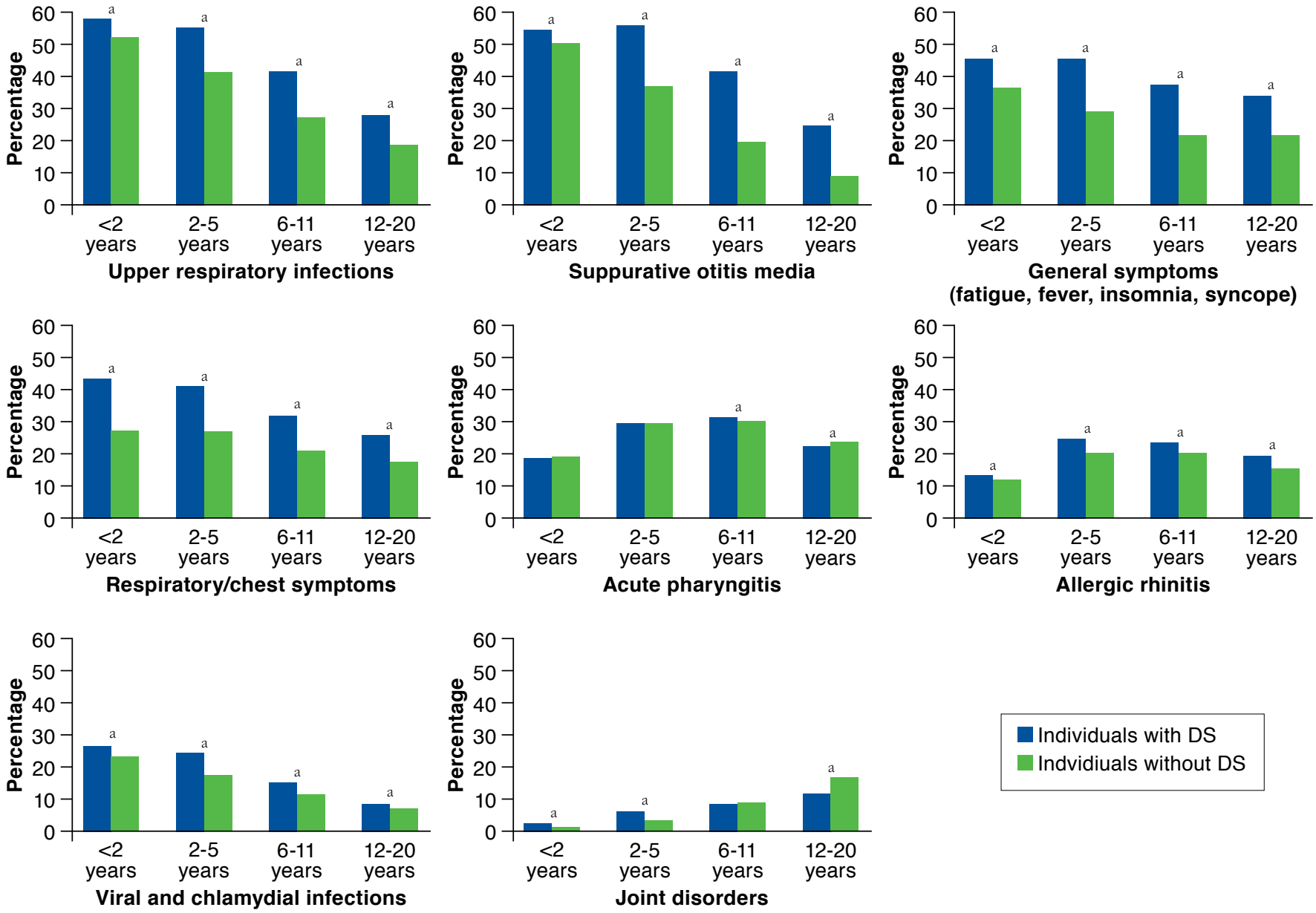

Individuals with DS

Indvidiuals without DS

aP values from chi-square tests for comparisons between individuals with and without DS within each age group for each condition were statistically significant ( $P<0.05)$. $P<0.001$ for all marked comparisons except for acute pharyngitis for ages $6-11$, where $P=0.008$, and ages 12-20, where $P=0.0001$; for allergic rhinitis for ages $<2$, where $P=0.025$; and for viral and chlamydial infection for ages $12-20$, where $P=0.001$

DS = Down syndrome.

gastrointestinal symptoms about twice as often among DS cases versus non-DS controls (Table 2).

Recently, Kageleiry et al. (2016) reported significantly higher out-of-pocket and third-party medical expenditures during childhood (birth to 18 years) among DS cases, compared with matched controls without chromosomal anomalies. ${ }^{19}$ In that claims-based study, total costs to families of patients aged over 18 years were over $\$ 18,000$ greater for DS cases, compared with controls, and insurer payments were more than $\$ 230,000$ greater for DS cases during the same time period. ${ }^{19}$ Although the current study does not report health care costs, Kageleiry et al.'s results are consistent with the significantly greater number and prevalence of comorbid conditions seen among DS cases in this analysis, which likely result in higher health care expenditures.
The prevalence of some diagnoses among DS cases in this study, however, were lower than expected based on previous work. For example, newborn screening for hearing problems is recommended (consistent with universal newborn screening guidelines) in infants with DS because of reported estimates of hearing loss from the AAP of up to $75 \%$ in this population. This study, however, showed that only up to $38 \%$ of DS cases had received a diagnosis of hearing loss during follow-up. Although this result is less than expected based on the AAP estimate, it is consistent with results from Roizen et al. (2014), in which $39 \%$ of parents indicated that their child with DS had been diagnosed with a hearing problem. ${ }^{9}$ A case series by Park et al. (2012) also reported estimates lower than the AAP, 
finding that $26.2 \%$ of infants failed their newborn hearing screenings. ${ }^{20}$ Discrepancies in prevalence may be due to lack of routine hearing testing, different data sources, or hearing loss not always being coded on claims.

Similarly, the AAP recommends that health care providers discuss with caregivers the potential for and symptoms of sleep disturbances in the first 6 months of life in children with DS, given estimates of $>50 \%$ prevalence of obstructive sleep apnea (OSA) in individuals with DS, particularly young children (aged approximately 2-5 years). ${ }^{6,21,22}$ In the current study, however, the 3-digit ICD-9-CM diagnosis of "organic sleep disorders" did not appear among the 25 most common diagnoses in DS cases aged $<12$ years. More specifically, OSA was diagnosed in only $8 \%-16 \%$ of DS cases, depending on cohort and age group, and "other sleep disorders" were diagnosed in only $1 \%-3 \%$ of DS cases (data not shown). The discrepancy between expected prevalence of sleep disturbances in individuals with DS and the frequency of such diagnoses observed in this study may point to a deficit in screening for these conditions in the DS population. It may also be a result of the underreporting of these conditions or undercoding on health care claims.

A strength of this study is the large sample size, representing nearly 28,000 individuals with DS and over 83,000 similar individuals without DS, as well as relatively large samples of cases and controls in the separate age groups. These samples included individuals with commercial and Medicaid health care coverage, representing individuals with diverse socioeconomic backgrounds. In addition, this study conducted direct comparisons of concomitant diagnoses between individuals with DS and non-DS individuals who were matched on important characteristics likely to be associated with the number and types of diagnoses received. This study design allowed for an estimate of the excess prevalence of comorbidities associated with a DS diagnosis.

\section{Limitations}

The limitations of this study include those inherent in any retrospective health care claims-based analysis. This study was limited to only those individuals with commercial health coverage or coverage by Medicaid. Consequently, results of this analysis may not be generalizable to individuals with other or additional insurance or those without health insurance coverage. There is also potential for misclassification of diagnoses or study outcomes, since individuals were identified through administrative claims data, which are subject to data coding limitations and data entry error.

Differences within age groups were not detected because ages were grouped together. For example, differences between individuals aged 2 and 3 years were not detected because in this analysis they were part of the 2-5 years age group. There may be systematic differences between the study cohorts that account for differences found in diagnoses. While matching cases and controls on important characteristics mitigated differences between cohorts, matching was limited to those characteristics that could be measured from administrative claims.
Finally, the process of matching cases to controls on specific variables, as well as the specific inclusion/exclusion criteria for selecting the study sample, may have resulted in the exclusion of individuals who may have had different outcomes from those selected for the study. Future research should build on recently published health care cost data among individuals with and without DS, specifically the financial impact of non-DS diagnoses, since differences in concomitant diagnoses may result in differences in health care costs between DS and non-DS individuals aged $<21$ years. Also, research should describe the average age when long-term conditions affecting individuals with DS are first diagnosed and categorize conditions as acute conditions versus chronic conditions because chronic conditions may have a larger effect clinically and financially.

\section{Conclusions}

This study shows that children and young adults with DS with either commercial or Medicaid insurance experience a substantially greater overall number and prevalence of many concomitant diagnoses, compared with similar individuals without DS. An overview is also offered of some of the specific diagnoses that are consistently more common among individuals with DS. Case managers may use these comorbidity measures to identify individuals in need of targeted quality-of-care management, and understanding the associated comorbidities of individuals with DS may be useful when forecasting expenditures and estimating potential savings provided by improved care management programs in this population. In addition, the differences in diagnosis patterns between commercially insured and Medicaidinsured individuals with DS may suggest differences in screening for various conditions between the 2 populations and/or real differences in the occurrence of comorbid conditions based on sociodemographic differences between the 2 cohorts. These results may warrant consideration by payers and case managers in order to provide better and equitable care for individuals with DS regardless of insurance plan and to be cognizant of the effect of concomitant diagnoses in that population.

\section{Authors}

AMANDA M. KONG, MPH, and KRISTIN A. EVANS, PhD, Truven Health Analytics, an IBM Company, Ann Arbor, Michigan. DANA HURLEY, PharmD, HUTH Global, Seattle, Washington; DIANA BRIXNER, PhD, University of Utah, Salt Lake City; CSILLA CSOBOTH, MD, PhD, Genentech, San Francisco, California; and JEANNIE VISOOTSAK, MD, F. Hoffman-LaRoche Pharmaceuticals, New York, New York.

AUTHOR CORRESPONDENCE: Diana Brixner, PhD, Department of Pharmacotherapy, University of Utah, 30 S. 2000 E., 4th Fl., Salt Lake City, UT 84112. Tel.: 801.581.3182;

E-mail: diana.brixner@utah.edu. 


\section{A Retrospective, Longitudinal, Claims-Based Comparison of Concomitant Diagnoses Between Individuals with and Without Down Syndrome}

\section{DISCLOSURES}

This study was sponsored and funded by Genentech. Truven Health Analytics, an IBM Company, receives payment from Genentech to conduct research, including the research for this study. Truven Health Analytics also receives payment from other pharmaceutical companies to conduct research. Kong and Evans are employed by Truven Health Analytics. Csoboth is employed by Genentech. Brixner reports fees paid to the University of Utah by Truven Health Analytics on her behalf for work related to this study. Hurley reports fees from Genentech for work on this study and for work outside of this study. At the time of this study, Visootsak was employed by F. HoffmanLaRoche Pharmaceuticals, parent company of Genentech.

All authors, including those affiliated with the study sponsor, were involved in the design of the study, interpretation of the data, writing of the manuscript, and the decision to submit the manuscript for publication. Study concept and design were contributed by Kong, Hurley, and Brixner, along with Evans. Kong and Evans collected the data, and data interpretation was performed by Csoboth, Visootsak, Brixner, and Hurley, with assistance from Kong. The manuscript was written by Evans, Kong, Hurley, and Brixner and revised by Kong, Hurley, Evans, and Brixner, with assistance from Csoboth and Visootsak.

\section{ACKNOWLEDGMENTS}

The authors thank Kavya Thelakkat (Truven Health Analytics) for assistance with medical writing.

\section{REFERENCES}

1. Ropers HH. Genetics of intellectual disability. Curr Opin Genet Dev. 2008;18(3)241-50.

2. Weijerman ME, de Winter JP. Clinical practice. The care of children with Down syndrome. Eur J Pediatr. 2010;169(2):1445-52.

3. Grieco J, Pulsifer M, Seligsohn K, Skotko B, Schwartz A. Down syndrome: cognitive and behavioral functioning across the lifespan. Am J Med Genet $C$ Semin Med Genet. 2015;169(2):135-49.

4. Head E, Lott IT, Wilcock D, Lemere CA. Aging in Down syndrome and the development of Alzheimer's disease neuropathology. Curr Alzheimer Res. 2016;13(1):18-29

5. Coppus AM. People with intellectual disabilities: what do we know about adulthood and life expectancy? Dev Disabil Res Rev. 2013;18(1):6-16.

6. Bull MJ, Saal HM, Braddock SR, et al. Clinical report-health supervision for children with Down syndrome. Pediatrics. 2011;128(2):393-406.

7. Schieve LA, Boulet SL, Boyle C, Rasmussen SA, Schendel D. Health of children 3 to 17 years of age with Down syndrome in the 1997-2005 National Health Interview Survey. Pediatrics. 2009;123(2):e253-e260.
8. Jiao T, Liou T, Young D, Brixner D. A comparison of demographic and clinical variables of Down syndrome patients in Utah and the United States. Value Health. 2013;16(7):A629 [Abstract PND65]. Available at: http://www. valueinhealthjournal.com/article/S1098-3015(13)03768-6/pdf. Accessed May 4, 2017.

9. Roizen NJ, Magyar CI, Kuschner ES, et al. A community cross-sectional survey of medical problems in 440 children with Down syndrome in New York State. J Pediatr. 2014;164(4):871-75.

10. Alexander M, Petri H, Ding Y, Wandel C, Khwaja O, Foskett N. Morbidity and medication in a large population of individuals with Down syndrome compared to the general population. Dev Med Child Neurol. 2016;58(3):246-54.

11. Stagliano DR, Nylund CM, Eide MB, Eberly MD. Children with Down syndrome are high-risk for severe respiratory syncytial virus disease. J Pediatr. 2015;166(3):703-09.

12. Ram G, Chinen J. Infections and immunodeficiency in Down syndrome. Clin Exp Immunol. 2011;164(1):9-16.

13. Weijerman ME, van Furth AM, van der Mooren MD, et al. Prevalence of congenital heart defects and persistent pulmonary hypertension of the neonate with Down syndrome. Eur J Pediatr. 2010;169(10):1195-99.

14. Kim MA, Lee YS, Yee NH, Choi JS, Choi JY, Seo K. Prevalence of congenital heart defects associated with Down syndrome in Korea. J Korean Med Sci. 2014;29(11):1544-49.

15. Stoll C, Dott B, Alembik Y, Roth MP. Associated congenital anomalies among cases with Down syndrome. Eur J Med Genet. 2015;58(12):674-80.

16. Bergstrom S, Carr H, Petersson G, et al. Trends in congenital heart defects in infants with Down syndrome. Pediatrics. 2016;138(1):e20160123.

17. Boulet SL, Molinari NA, Grosse SD, Honein MA, Correa-Villasenor A. Health care expenditures for infants and young children with Down syndrome in a privately insured population. J Pediatr. 2008;153(2):241-46.

18. Zhu JL, Hasle H, Correa A, et al. Hospitalizations among persons with Down syndrome: a national cohort study in Denmark. Am J Med Genet A. 2013;0(4):650-57.

19. Kageleiry A, Samuelson D, Duh MS, Lefebvre P, Campbell J. Out-ofpocket medical costs and third-party healthcare costs for children with Down syndrome. Am J Med Genet A. 2017;173(3):627-37.

20. Park AH, Wilson MA, Stevens PT, Harward R, Hohler N. Identification of hearing loss in pediatric patients with Down syndrome. Otolaryngol Head Neck Surg. 2012;146(1):135-40.

21. Shott SR, Amin R, Chini B, Heubi BS, Hotze S, Akers R. Obstructive sleep apnea: should all children with Down syndrome be tested? Arch Otolaryngol Head Neck Surg. 2006;132(4):432-36.

22. Fitzgerald DA, Paul A, Richmond C. Severity of obstructive apnoea in children with Down syndrome who snore. Arch Dis Child. 2007;92(5):423-25. 
A Retrospective, Longitudinal, Claims-Based Comparison of Concomitant Diagnoses Between Individuals with and Without Down Syndrome

\section{APPENDIX A Patient Attrition}

Individuals with a diagnosis code for Down syndrome

(ICD-9-CM 758.0x) January 1, 2008-December 31, 2014

(earliest date $=$ index date)

Commercial, $\mathrm{N}=47,791$

Medicaid, $\mathrm{N}=34,913$

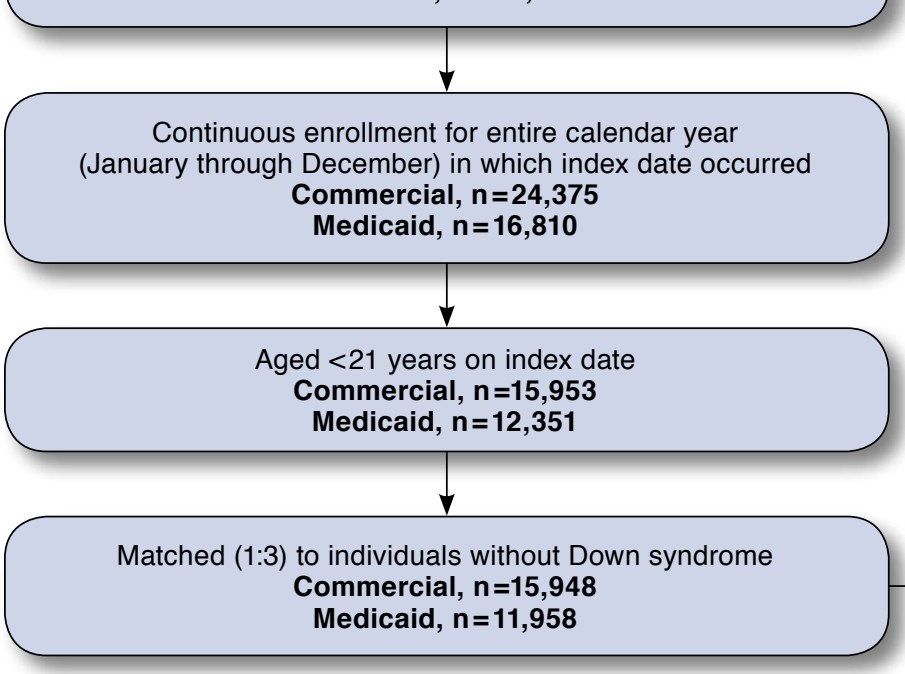

Individuals without a diagnosis of Down syndrome or another chromosomal anomaly (ICD-9-CM 758.xx)

Commercial, $n=99,280,768$

Medicaid, $n=12,672,744$

ICD-9-CM = International Classification of Diseases, Ninth Revision, Clinical Modification. 


\section{A Retrospective, Longitudinal, Claims-Based Comparison of Concomitant Diagnoses Between Individuals with and Without Down Syndrome}

\section{APPENDIX B Number and Proportion of All Individuals with and Without Down Syndrome (Commercial and Medicaid Combined) with the 10 Most Common 3-Digit ICD-9-CM Diagnoses Among Individuals Without Down Syndrome in Each Age Group}

Most Common ICD-9-CM Diagnoses Among Controls During Follow-upa

\section{Aged $<2$ Years}

Acute upper respiratory infections of multiple or unspecified sites (465.xx) Suppurative and unspecified otitis media (382.xx)

General symptoms (e.g., fatigue, fever, insomnia, syncope; 780.xx)

Symptoms involving respiratory system and other chest symptoms (786.xx)

Viral and chlamydial infections (079.xx)

Symptoms involving digestive system (787.xx)

Acute bronchitis and bronchiolitis (466.xx)

Nonsuppurative otitis media and Eustachian tube disorders (381.xx)

Acute pharyngitis (462.xx)

Disorders of conjunctiva (372.xx)

Atopic dermatitis and related conditions (691.xx)

Aged 2-5 Years

Acute upper respiratory infections of multiple or unspecified sites (465.xx)

Suppurative and unspecified otitis media (382.xx)

Acute pharyngitis (462.xx)

General symptoms (e.g., fatigue, fever, insomnia, syncope; 780.xx)

Symptoms involving respiratory system and other chest symptoms (786.xx)

Allergic rhinitis (477.xx)

Viral and chlamydial infections (079.xx)

Streptococcal sore throat and scarlet fever (034.xx)

Disorders of conjunctiva (372.xx)

Symptoms involving digestive system (787.xx)

Acute sinusitis (461.xx)

Asthma (493.xx)

Specific delays in development (315.xx)

\section{Aged 6-11 Years}

Acute pharyngitis (462.xx)

Acute upper respiratory infections of multiple or unspecified sites (465.xx)

General symptoms (e.g., fatigue, fever, insomnia, syncope; 780.xx)

Symptoms involving respiratory system and other chest symptoms (786.xx)

Allergic rhinitis (477.xx)

Suppurative and unspecified otitis media (382.xx)

Streptococcal sore throat and scarlet fever (034.xx)

Disorders of refraction and accommodation (367.xx)

Hyperkinetic syndrome of childhood (314.xx)

Asthma (493.xx)

Acute sinusitis (461.xx)

Other symptoms involving abdomen and pelvis (789.xx)

\section{Aged 12-20 Years}

Acute pharyngitis (462.xx)

General symptoms (e.g., fatigue, fever, insomnia, syncope; 780.xx)

Acute upper respiratory infections of multiple or unspecified sites (465.xx)

Symptoms involving respiratory system and other chest symptoms (786.xx)

Other and unspecified disorders of joint (719.xx)

Other symptoms involving abdomen and pelvis (789.xx)

\begin{tabular}{|c|c|c|c|c|}
\hline \multicolumn{2}{|c|}{ Cases $^{\mathrm{b}}$} & \multicolumn{2}{|c|}{ Controls ${ }^{\mathrm{c}}$} & $\begin{array}{l}P \text { Values from } \\
\text { Chi-Square Test }\end{array}$ \\
\hline \multicolumn{2}{|c|}{$\mathrm{n}=4,227$} & \multicolumn{2}{|c|}{$\mathrm{n}=12,661$} & \\
\hline$\%$ & $\mathbf{n}$ & $\%$ & $\mathrm{n}$ & \\
\hline 59.1 & $(2,499)$ & 52.9 & $(6,705)$ & $<0.001$ \\
\hline 55.1 & $(2,327)$ & 51.2 & $(6,485)$ & $<0.001$ \\
\hline 46.8 & $(1,980)$ & 37.2 & $(4,709)$ & $<0.001$ \\
\hline 44.9 & $(1,899)$ & 28.6 & $(3,625)$ & $<0.001$ \\
\hline 26.6 & $(1,125)$ & 23.5 & $(2,981)$ & $<0.001$ \\
\hline 37.1 & $(1,567)$ & 20.8 & $(2,636)$ & $<0.001$ \\
\hline 32.0 & $(1,352)$ & 19.6 & $(2,487)$ & $<0.001$ \\
\hline 44.7 & $(1,889)$ & 19.2 & $(2,434)$ & $<0.001$ \\
\hline 18.7 & $(791)$ & 19.2 & $(2,426)$ & 0.521 \\
\hline 24.4 & $(1,031)$ & 17.1 & $(2,164)$ & $<0.001$ \\
\hline 13.2 & $(556)$ & 16.8 & $(2,132)$ & $<0.001$ \\
\hline \multicolumn{2}{|c|}{$\mathbf{n}=8,955$} & \multicolumn{2}{|c|}{$\mathrm{n}=26,865$} & \\
\hline$\%$ & $\mathrm{n}$ & $\%$ & $\mathbf{n}$ & \\
\hline 56.0 & $(5,012)$ & 42.0 & $(11,272)$ & $<0.001$ \\
\hline 56.8 & $(5,089)$ & 37.5 & $(10,081)$ & $<0.001$ \\
\hline 30.1 & $(2,693)$ & 30.1 & $(8,077)$ & 0.989 \\
\hline 47.2 & $(4,230)$ & 29.9 & $(8,029)$ & $<0.001$ \\
\hline 41.9 & $(3,755)$ & 28.1 & $(7,539)$ & $<0.001$ \\
\hline 24.3 & $(2,180)$ & 20.0 & $(5,373)$ & $<0.001$ \\
\hline 24.2 & $(2,170)$ & 17.7 & $(4,743)$ & $<0.001$ \\
\hline 22.8 & $(2,038)$ & 17.6 & $(4,728)$ & $<0.001$ \\
\hline 31.0 & $(2,772)$ & 16.3 & $(4,383)$ & $<0.001$ \\
\hline 36.4 & $(3,257)$ & 16.2 & $(4,354)$ & $<0.001$ \\
\hline 37.8 & $(3,385)$ & 16.0 & $(4,292)$ & $<0.001$ \\
\hline 25.0 & $(2,235)$ & 15.6 & $(4,187)$ & $<0.001$ \\
\hline 52.8 & $(4,728)$ & 10.9 & $(2,929)$ & $<0.001$ \\
\hline \multicolumn{2}{|c|}{$\mathrm{n}=11,810$} & \multicolumn{2}{|c|}{$\mathrm{n}=35,430$} & \\
\hline$\%$ & $\mathrm{n}$ & $\%$ & n & \\
\hline 31.8 & $(3,757)$ & 30.5 & $(10,808)$ & 0.008 \\
\hline 42.4 & $(5,003)$ & 27.4 & $(9,719)$ & $<0.001$ \\
\hline 38.3 & $(4,525)$ & 22.1 & $(7,829)$ & $<0.001$ \\
\hline 32.7 & $(3,860)$ & 21.6 & $(7,646)$ & $<0.001$ \\
\hline 23.5 & $(2,779)$ & 20.7 & $(7,331)$ & $<0.001$ \\
\hline 42.4 & $(5,012)$ & 19.6 & $(6,939)$ & $<0.001$ \\
\hline 22.1 & $(2,611)$ & 16.8 & $(5,968)$ & $<0.001$ \\
\hline 27.1 & $(3,198)$ & 15.4 & $(5,449)$ & $<0.001$ \\
\hline 12.9 & $(1,519)$ & 15.0 & $(5,309)$ & $<0.001$ \\
\hline 20.1 & $(2,374)$ & 14.8 & $(5,249)$ & $<0.001$ \\
\hline 34.9 & $(4,116)$ & 14.4 & $(5,090)$ & $<0.001$ \\
\hline 14.2 & $(1,681)$ & 14.2 & $(5,046)$ & 0.982 \\
\hline \multicolumn{2}{|c|}{$\mathrm{n}=11,984$} & \multicolumn{2}{|c|}{$\mathbf{n}=35,952$} & \\
\hline$\%$ & $\mathrm{n}$ & $\%$ & $\mathbf{n}$ & \\
\hline 22.5 & $(2,697)$ & 24.1 & $(8,647)$ & 0.001 \\
\hline 35.1 & $(4,209)$ & 22.1 & $(7,932)$ & $<0.001$ \\
\hline 28.9 & $(3,462)$ & 19.5 & $(7,018)$ & $<0.001$ \\
\hline 26.9 & $(3,219)$ & 18.4 & $(6,613)$ & $<0.001$ \\
\hline 11.6 & $(1,396)$ & 16.6 & $(5,976)$ & $<0.001$ \\
\hline 15.1 & $(1,815)$ & 15.6 & $(5,626)$ & 0.187 \\
\hline
\end{tabular}

continued on next page 
APPENDIX B Number and Proportion of All Individuals with and Without Down Syndrome (Commercial and Medicaid Combined) with the 10 Most Common 3-Digit ICD-9-CM Diagnoses Among Individuals Without Down Syndrome in Each Age Group (continued)

\begin{tabular}{|c|c|c|c|c|c|}
\hline Most Common ICD-9-CM Diagnoses Among Controls During Follow-up ${ }^{a}$ & \multirow{2}{*}{\multicolumn{2}{|c|}{$\begin{array}{c}\text { Cases }^{\mathrm{b}} \\
\mathrm{n}=11,984\end{array}$}} & \multirow{2}{*}{\multicolumn{2}{|c|}{$\begin{array}{l}\text { Controls }^{\mathrm{c}} \\
\mathrm{n}=35,952 \\
\end{array}$}} & \multirow[t]{3}{*}{$\begin{array}{l}P \text { Values from } \\
\text { Chi-Square Test }\end{array}$} \\
\hline \multirow{2}{*}{ Aged 12-20 Years } & & & & & \\
\hline & $\%$ & n & $\%$ & n & \\
\hline Allergic rhinitis (477.xx) & 19.9 & $(2,379)$ & 15.3 & $(5,508)$ & $<0.001$ \\
\hline Symptoms involving head and neck (784.xx) & 15.7 & $(1,882)$ & 15.3 & $(5,506)$ & 0.307 \\
\hline Acute sinusitis (461.xx) & 26.1 & $(3,124)$ & 15.1 & $(5,418)$ & $<0.001$ \\
\hline Diseases of sebaceous glands (706.xx) & 12.5 & $(1,503)$ & 14.4 & $(5,175)$ & $<0.001$ \\
\hline Disorders of refraction and accommodation (367.xx) & 21.5 & $(2,571)$ & 13.4 & $(4,812)$ & $<0.001$ \\
\hline Symptoms involving digestive system (787.xx) & 19.4 & $(2,321)$ & 12.4 & $(4,463)$ & $<0.001$ \\
\hline \multicolumn{6}{|c|}{$\begin{array}{l}{ }^{a} \text { More than } 10 \text { diagnoses on each list because of differences in top } 10 \text { diagnoses between commercial and Medicaid; all unique diagnoses in the top } 10 \text { for each age group in } \\
\text { each cohort are listed. } \\
{ }^{b} \text { Cases=with DS. } \\
\text { cControls=without DS. } \\
\text { DS = Down syndrome; ICD-9-CM = International Classification of Diseases, Ninth Revision, Clinical Modification. }\end{array}$} \\
\hline
\end{tabular}

